\title{
A Survey on How Ocular Surface Demodex Infestation Interactively Associates with Diabetes Mellitus and Dry Eye Disease
}

\author{
Chang Huang ${ }^{1}$. Shuze Chen ${ }^{2}$. Sheng $\mathrm{Fu}^{3} \cdot$ Yingli $\mathrm{Li}^{4} \cdot \mathrm{Zhhenhao}^{\mathrm{Li}} \mathrm{i}^{5} \cdot$ Siqi $\mathrm{Li}^{6} \cdot$ Xiaoqian Liang $^{1} \cdot \mathrm{Zihong} \mathrm{Wang}^{1}$. \\ Zhoucheng Wang ${ }^{1} \cdot$ Yifan Chen ${ }^{1}$. Qixin Deng ${ }^{1}$. Guoguo $\mathrm{Yi}^{7}$ (D) Min Fu ${ }^{8}$
}

Received: 1 August 2020 / Accepted: 23 March 2021 / Published online: 3 April 2021

(c) The Author(s) 2021

\begin{abstract}
Purpose Prevention of ocular surface (OS) Demodex infestation plays an important role in OS hygiene and variety of factors may be associated with it, in which diabetes mellitus (DM) or dry eye disease (DED) has caught the attention of most scholars. However, there has been no research on whether there was a potential interaction between DM and DED in the process of OS Demodex infestation. This cross-sectional study was implemented in Zhujiang Hospital of Southern Medical University. Methods Ophthalmologic interviews, questionnaires, and examinations were conducted. Factors including general information, DM status, dry eye condition, etc. were collected to study the correlation of DM and DED on OS Demodex infestation. Results After statistical analysis, we found that both DM $(P<0.001)$ and DED $(P=0.013<0.05)$ are closely associated with OS Demodex infestation. Compared with DED, DM has higher priority association with OS Demodex infestation, and patients with both diseases have a significant higher risk of OS Demodex infestation $(R=0.197, P<0.001)$. Meanwhile, age $(R=0.299, P<0.001)$ and hypertension $(P<0.05)$ were also correlated with OS Demodex infestation.
\end{abstract}

Conclusion This study provides a new evidence-based basis for clinical prevention and management of OS Demodex infestation.

Keywords Ocular surface Demodex infestation · Diabetes mellitus · Dry eye disease $\cdot$ Cross-sectional study

\section{Introduction}

Ocular surface (OS) Demodex infestation is a common disease in population [1, 2]. Demodex (Acariformes:Prostigmata) is one of the most common

Chang Huang, Shuze Chen, Sheng Fu, and Yingli Li contributed equally.

Guoguo Yi

yigg@mail.sysu.edu.cn

$\triangle$ Min Fu

min_fu1212@163.com

1 The Second Clinical School, Southern Medical University, Guangzhou, Guangdong, China

2 Department of Gastroenterology, Nanfang Hospital, Southern Medical University, Guangzhou, Guangdong, China

3 The University of South China, Hengyang, Hunan, China

4 Department of Ophthalmology, Shenzhen Hospital, Southern Medical University, 1333 Xinhu Road, Baoan District,

Shenzhen, China ectoparasites in humans [3]. Among many species of Demodex, only Demodex folliculorum and Demodex brevis Akbulatova are found in humans, including the face, cheeks, forehead, nose, and eyelids [4, 5]. Eyelid, follicular mycosis lives in ciliated sacs. Besides, short mold lives in eyelid, eyelid gland and sebaceous gland [6]. They eat skin cells, hormones and oils from hair follicles [7]. The pathogenic role of Demodex in ophthalmology has not been controversial [8].

5 Department of General Surgery, Nanfang Hospital, Southern Medical University, Guangzhou, Guangdong, China

6 Department of Pediatrics, Zhujiang Hospital, Southern Medical University, Guangzhou, Guangdong, China

7 Department of Ophthalmology, The Sixth Affiliated Hospital of Sun Yat-Sen University, Guangzhou, Guangdong, China

8 Department of Ophthalmology, Zhujiang Hospital, Southern Medical University, No. 253 Industrial Avenue Center, Haizhu District, Guangzhou, Guangdong, China 
For some, it is a contributing factor, for others it has no obvious symptoms. This indicates that an individual's response depends on a number of factors. Some studies have shown that Demodex is a non-pathogenic parasite [9]. However, the other reports suggest that OS Demodex infestation was a critical factor in many eye diseases $[1,10]$, such as nodular corneal scars, blepharitis, conjunctivitis and trichiasis. Thus, the prevention of OS Demodex infestation plays an important role in ocular surface hygiene. It has been found that multitudinous factors may affect OS Demodex infestation, such as age, diabetes mellitus (DM) [11], dry eye disease (DED) [12], and meibomian gland dysfunction (MGD) [13], etc.

DM is a metabolic disease characterized by hyperglycemia which can lead to chronic damage and dysfunction of various tissues [14]. Previous studys showed that Demodex follicle sacs were more common in diabetic patients than in healthy volunteers $[15,16]$. Beside, DED is a disorder of the tear film, which is a highly prevalent chronic multifactorial disease, causing an itchy eyes or foreign body sensation. Many reports [17] suggest that OS Demodex infestation is prevalent in DED patients. It had been reported that Demodex folliculorum showed a high prevalence in patients with DED [17, 18]. Meanwhile, another study indicated that Demodex were implicated in DED syndrome [19].

To futher understand whether there was an interaction between DM and DED in the process of OS Demodex infestation, we designed a cross-sectional study to explore the interaction between DM and DED in OS Demodex infestation. Hence, we can provide an evidence-based medicine for the future prevention and treatment of ocular surface Demodex infestation.

\section{Materials and Methods}

This cross-sectional study was conducted from June 2018 to October 2018 at the Zhujiang Hospital of Southern Medical University in China. The research has been registered on Chinese clinical trial registry (ChiCTR1800016357). The study was performed in accordance with principles of the Declaration of Helsinki, the International Conference on Harmonisation, Good Clinical Practice guidelines, and all applicable laws and regulations. The study protocol and one amendment to the protocol were reviewed and approved by the Zhujiang Hospital Human Experimental Committee, and all patients provided written informed consent prior to starting study treatment.

\section{Participants}

This study recruited 255 outpatients and inpatients from Zhujiang Hospital of Southern Medical University. The exclusion criteria were as follows: recent acute complications and infestations such as diabetic ketoacidosis; history of eye surgery and trauma within 6 months; cornea contact lens wear history; elevated blood glucose caused by Type I DM and other causes; dry eye caused by rheumatic immune disease and hyperthyroidism; pregnancy, lactating female; and mentally ill patients. Ophthalmologic interviews, questionnaires and examinations were performed to all participants. Data of all participants included general information (age, sex, weight, height, and history of other diseases such heart disease), routine blood test, hypertension, etc. To facilitate the analysis of age, we divide the age into several stages: 0-9, 10-19, 20-29, 30-39, 40-49, 50-59, 60-69, $70-79$, and $80-89$. The outcome variables were ocular surface disease index (OSDI) symptom questionnaire, tear film break-up time, eyelid symptom score, total cholesterol, fasting blood glucose, fasting insulin, HbA1c and so on. Outcomes were cross-sectionally conducted on factors associated with OS Demodex infestation, including D. folliculorum and D. brevis.

\section{Assessment of Ocular Surface Demodex Infestation}

Two lashes were removed from each eyelid by fine forceps under a slit-lamp microscope for each patient. The removed lashes from each eyelid were placed separately on a glass slide. OS Demodex detection and counting was performed by a professional technician who did not know each patient's information. Under a light microscope, 1 drop of saline or fluorescein-containing solution was applied by a pipette to the edge of the coverslip before counting.

The diagnosis of ocular surface Demodex infestation is based on the 2018 expert consensus on diagnosis and treatment of Demodex blepharitis China diagnostic criteria: (1) Demodex in all phases are counted; (2) adult patients have a Demodex count of $3 / 3$ eyelashes in any of the four eyelids; (3) less than the above criteria is suspected positive, combined with clinical manifestations; if necessary, other pathogenic microorganisms can be examined at the same time, such as bacteria and fungi. Therefore, the severity of OS Demodex infestation is divided into 3 level: negative, suspicious positive and positive. 


\section{Assessment of Diabetes Mellitus}

Diabetes mellitus was diagnosed according to the 2018 US ADA diagnostic criteria: (1) glycosylated hemoglobin $\mathrm{HbA} 1 \mathrm{c} \geq 6.5 \%$. (2) Fasting blood glucose $\mathrm{FPG} \geq 7.0 \mathrm{mmol} / \mathrm{L}$. Fasting is defined as no calorie intake for at least $8 \mathrm{~h}$. (3) In the oral glucose tolerance test, the blood glucose was $\geq 11.1 \mathrm{mmol} / \mathrm{L}$ for $2 \mathrm{~h}$. (4) In patients with typical hyperglycemia or hyperglycemia crisis, the random blood glucose was $\geq 11.1 \mathrm{mmol} / \mathrm{L}$. The duration of DM was divided into the following stages: 0 years, $\leq 5$ years, $\leq 10$ years, and $\geq 10$ years.

\section{Assessment of Dry Eye Outcomes}

Based on American Academy of Ophthalmology and DEWS, the dry eye patients was define as individuals who had at least one of the classical symptoms plus one or more alterations in the objective tests analyzed. They were evaluated for common symptoms of dry eye ocular discomfort including: soreness, gritty sensation, itchiness, redness, blurred vision that improves with blinking, and excessive tearing. Both the frequency of these symptoms were based on criteria proposed by the American Academy of Ophthalmology and DEWS.

To analyze the severity of DED, we divide the OSDI scores into 4 degrees: no obvious symptoms: OSDI scores $\leq 12.0$. Mild: $12.00<$ scores $\leq 22.0$. Moderate: $22.0<$ scores $\leq 32.0$. Severe: $32.0<$ scores $\leq 100$. The main points of comparison were subjective complaints, objective findings on corneal staining with fluorescein, meibomian glands and lid alterations and break-up time (BUT), basal secretion test, impression cytology of the conjunctival injection, and conjunctival staining. Subjects also underwent objective clinical assessment for DED.

\section{Statistical Analysis}

Data were analyzed with the Statistical Package for Social Sciences version 24.0 software (SPSS Inc, Chicago, IL, USA). The descriptive statistics were presented as mean \pm SD for normally distributed continuous variables. Nonnumerical data were recorded as presence (yes) or absence (no). Categorical variables were compared by using the Chi-square or Fisher exact test; continuous variables, using the one-way analysis of variance (ANOVA), Kruskal-Wallis test, and Jonckheere-Terpstra test. The Pearson Chi-square test and Fisher's exact test were used for the rest of comparative and correlative analyses. Non-parametric Spearmen's correlation tests were used to determine differences between ocular surface Demodex infestation and other risk factors, and age-group analysis was performed using the Mantel-Haenszel test. Finally, Pearson correlation test and Mantel-Haenszel test were used to analyze the interaction between DM and DED in ocular Demodex infestation when graded. $P<0.05$ was considered statistically significant in all analyses.

\section{Results}

\section{Participants and the Basic Clinical Information}

After applying all entry criteria, 444 eyes from 255 participants (aged 18-84) were included in the analysis. The missing data in the study are processed by the adjacent point interpolation method in SPSS software. Mean age was 53.96 (standard deviation [SD], 16.148) years. Overall, $45.1 \%$ of the participants were male, while $54.9 \%$ were female. Total OS Demodex infestation results were $23.4 \%$ for positive, $33.3 \%$ for suspicious positive, and $43.3 \%$ for negative.

In participants, there was a significant correlation between age and OS Demodex infestation $(P<0.001)$. We also found that OS Demodex infestation was strongly associated with MGD $(P=0.002)$, DED $(P=0.013)$, and OSDI scores $(P=0.030)$. Both DM $(P<0.001)$ and DM duration $(P<0.001)$ had significant correlation with OS Demodex infestation as well. Moreover, the prevalence of OS Demodex infestation was much higher in participants with hypertension $(P=0.016)$.

However, no difference was exhibited between sex and OS Demodex infestation $(P>0.05)$, or between hyperlipidemia and OS Demodex infestation $(P>0.05)$. Further analysis of hematological parameters showed there was no difference between HbA1C, HDL, LDL, TG, TC, and OS Demodex infestation (Table 1; Fig. 1).

\section{The Relationship Between OS Demodex Infestation with DM and DED}

From Table 1, both DM and DED had a significant correlation with OS Demodex infestation. We further divided DM into two layers (yes or no), and further statistically analyzed DED and OS Demodex infestation in each layer (Table 2), with results suggesting that there was no significant difference between DED and OS Demodex infestation in diabetic patients $(P=0.316)$, while in non-diabetic patients a difference between the latter two was distinguished $(P=0.017)$. Similarly, according to DED (yes or no), further statistical analysis of DM and OS Demodex infestation in each layer (Table 2) showed significant correlations between DM (yes or no) and OS Demodex infestation in patients with DED (DM: $P=0.015$; Non-DM: $P=0.004$ ).

In short, both DM and DED had an nexus with OS Demodex infestation, and the association between DM and 
Table 1 Baseline characteristics of the participants, according to the infestation of OS Demodex

\begin{tabular}{|c|c|c|c|c|}
\hline Characteristic & Negative & Suspicious positive & Positive & $P$ value \\
\hline Total $n(\%)$ & $192(43.2)$ & $148(33.3)$ & $104(23.4)$ & - \\
\hline Age, (years) & $49.84 \pm 17.76$ & $54.79 \pm 4.13$ & $62.12 \pm 12.81$ & $<0.001^{\mathrm{a}}$ \\
\hline \multicolumn{5}{|l|}{ Sex, $(\%)$} \\
\hline Male & 41.6 & 35.0 & 23.4 & \multirow[t]{2}{*}{$0.771^{\mathrm{b}}$} \\
\hline Female & 44.5 & 32.0 & 23.5 & \\
\hline \multicolumn{5}{|l|}{ MGD, (\%) } \\
\hline Yes & 40.8 & 36.7 & 22.4 & \multirow[t]{2}{*}{$0.002^{\mathrm{b}}$} \\
\hline No & 62.3 & 21.8 & 15.8 & \\
\hline \multicolumn{5}{|l|}{$\mathrm{DM},(\%)$} \\
\hline Yes & 37.9 & 28.0 & 34.1 & \multirow[t]{2}{*}{$<0.001^{\mathrm{b}}$} \\
\hline No & 49.1 & 37.5 & 13.4 & \\
\hline \multicolumn{5}{|l|}{$\mathrm{DED},(\%)$} \\
\hline Yes & 35.5 & 38.1 & 26.3 & \multirow[t]{2}{*}{$0.013^{\mathrm{b}}$} \\
\hline No & 50.2 & 29.7 & 20.1 & \\
\hline \multicolumn{5}{|l|}{ Hypertension, (\%) } \\
\hline Yes & 33.1 & 38.0 & 28.9 & \multirow[t]{2}{*}{$0.016^{\mathrm{b}}$} \\
\hline No & 48.2 & 30.7 & 21.1 & \\
\hline \multicolumn{5}{|l|}{ Hyperlipidemia, (\%) } \\
\hline Yes & 41.9 & 32.4 & 25.7 & \multirow[t]{2}{*}{$0.803^{\mathrm{b}}$} \\
\hline No & 44.4 & 32.9 & 22.7 & \\
\hline DM duration (years) & $2.56 \pm 5.51$ & $3.10 \pm 5.91$ & $6.34 \pm 6.68$ & $<0.001^{\mathrm{a}}$ \\
\hline $\operatorname{HbA1C}(\%)$ & $8.22 \pm 2.89$ & $8.26 \pm 2.33$ & $8.34 \pm 2.72$ & $0.586^{\mathrm{a}}$ \\
\hline OSDI score & $11.63 \pm 11.23$ & $14.88 \pm 14.43$ & $16.16 \pm 16.65$ & $0.030^{\mathrm{a}}$ \\
\hline HDL (mg/dl) & $1.27 \pm 0.43$ & $1.18 \pm 0.29$ & $1.32 \pm 0.35$ & $0.338^{\mathrm{a}}$ \\
\hline $\mathrm{LDL}(\mathrm{mg} / \mathrm{dl})$ & $3.22 \pm 0.96$ & $2.86 \pm 0.90$ & $3.39 \pm 1.24$ & $0.681^{\mathrm{a}}$ \\
\hline $\mathrm{TG}(\mathrm{mg} / \mathrm{dl})$ & $1.89 \pm 1.91$ & $1.96 \pm 1.21$ & $1.66 \pm 1.26$ & $0.927^{\mathrm{a}}$ \\
\hline $\mathrm{TC}(\mathrm{mg} / \mathrm{dl})$ & $5.22 \pm 2.27$ & $4.90 \pm 1.26$ & $5.67 \pm 2.59$ & $0.173^{\mathrm{a}}$ \\
\hline
\end{tabular}

$D M$ Diabetes mellitus, $D E D$ dry eye disease, $M G D$ meibomian gland dysfunction, $H b A 1 C$ glycosylated hemoglobin, OSDI score Ocular Surface Disease Index score, $H D L$ high-density lipoprotein, $L D L$ low-density lipoprotein, $T G$ triglycerides, $T C$ serum total cholesterol

a Jonckheere-Terpstra test

${ }^{\mathrm{b}} \mathrm{Chi}$-square test
OS Demodex infestation was greater than DED. In other words, as long as the participants had diabetes, whether he/ she had dry eye disease was insignificant for OS Demodex infestation.

Moreover, we divided all participants into four levels according to whether the patient had DM and DED: (1) DM- and DED- for the first level. (2) DM- and DED + for the second level. (3) DM + and DED- for the third level. (4) $\mathrm{DM}+$ and DED + for the fourth level. The prevalence and the statistical analysis of each group were shown in Table 3.

From grade 1 to grade 4, it was indicated that the results of OS Demodex infestation was becoming increasingly serious, and there was a significant linear positive correlation between them $(\mathrm{M}-\mathrm{H}$ test: $P<0.001$; Pearson coefficient: $R=0.197, P<0.001)$. It indicated that both DM and DED were not only related to ocular surface Demodex infestation, but also played a synergistic role, which exacerbated the infestation of ocular surface Demodex together.

\section{Statistical Inference of Linear Trends Between Various Basic Indicators and Locust Infestation}

In addition, Spearmen's correlation was used to analyze whether there was a significant linear correlation between ocular surface Demodex infestation and age $(\rho=0.265$, $P<0.001)$, DM $(\rho=0.189, P<0.001)$, DM duration $(\rho=0.256, P<0.001)$. Also, there was a correlation between ocular surface Demodex infestation and MGD $(\rho=0.184$, $P<0.001)$, DED $(\rho=0.138, P=0.006<0.01)$, hypertension $(\rho=0.134, P=0.05<0.01)$.

From Table 1, we found an apparent correlation between age and OS Demodex infestation. After further grading the age, the severity of OS Demodex infestation is higher when 

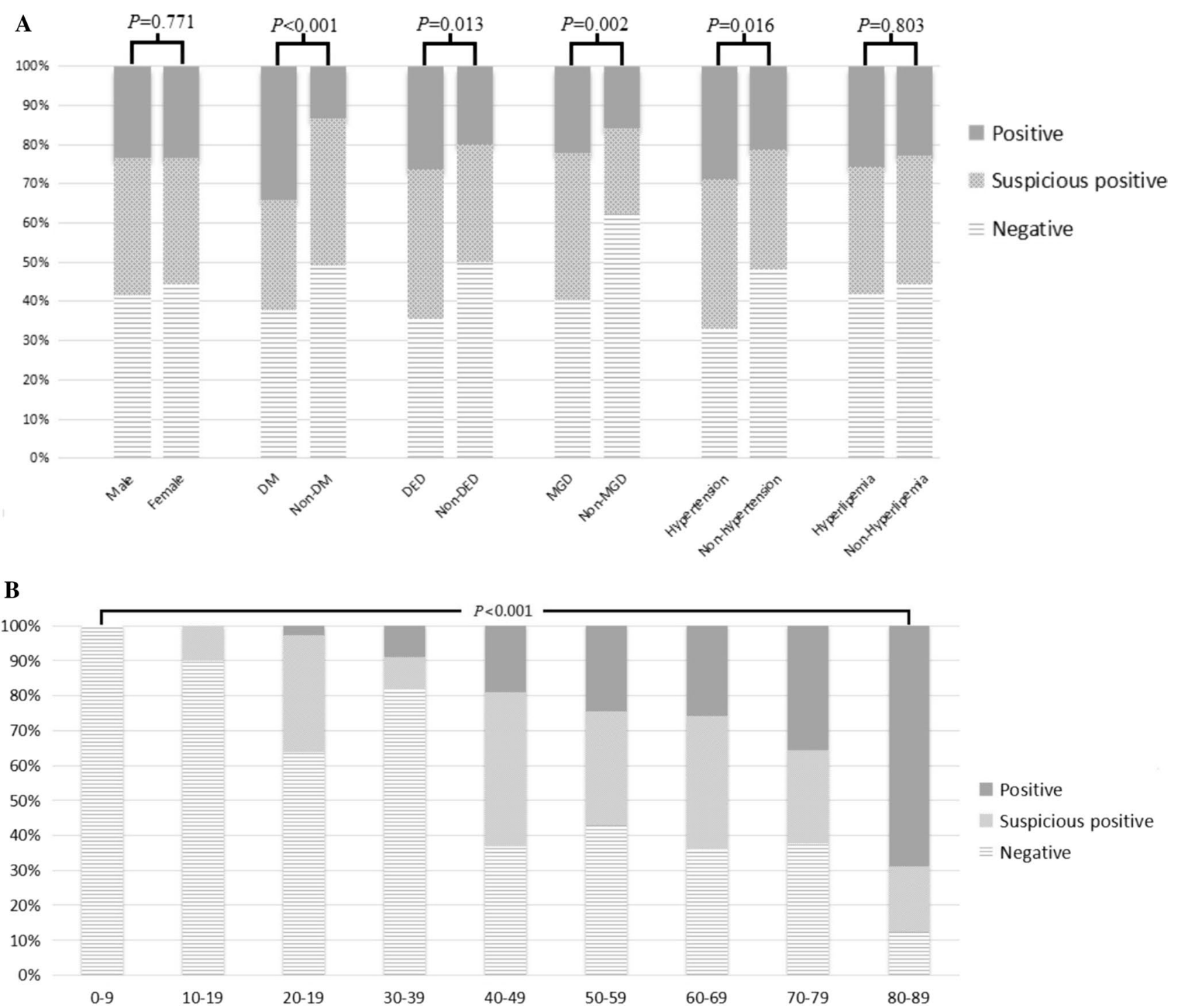

Fig. 1 Differences between OS Demodex infestation and sex, DED, DM, hypertension, hyperlipidemia, MGD and age. a The relationship between ocular surface Demodex infestation and sex, diabetes mellitus, dry eye disease, meibomian gland dysfunction, hypertension and hyperlipidemia. After Chi-square test, diabetes mellitus, dry eye disease, tarsal gland dysfunction and hypertension were correlated with ocular surface Demodex infestation, but there was no statistical difference between sexes and hyperlipidemia. b The relationship between ocular surface Demodex infestation and age. After Jonckheere-Terpstra test, there was an apparent correlation between age and Demodex infestation, and a looming linear trend was also observed in the figure

correlated $(R=0.069, P>0.05)$. It can be seen that with the increase of age, the infestation of OS Demodex gradually deteriorates, and as the length of DM is prolonged, the infestation of OS Demodex was also aggravated.

\section{Discussion}

Demodex, a small parasitic mite that affects mammals, was first identified in 1841 [3]. However, only recently attracted the attention of clinicians, including ophthalmologists, dermatologists and other specialists [20] Demodex infestation tation, while the OSDI score was not significantly linearly 
Table 2 Relationship between DM, DED, and OS Demodex infestation

\begin{tabular}{|c|c|c|c|c|c|}
\hline$\overline{\mathrm{DM}^{\mathrm{a}}}$ & DED & Negative (\%) & Suspicious positive (\%) & Positive (\%) & $P$ value \\
\hline \multirow[t]{2}{*}{ Yes } & Yes & 34.5 & 29.9 & 35.6 & 0.316 \\
\hline & No & 45.0 & 26.1 & 28.8 & \\
\hline \multirow[t]{2}{*}{ No } & Yes & 36.4 & 45.5 & 18.2 & 0.017 \\
\hline & No & 56.1 & 33.7 & 10.2 & \\
\hline $\mathrm{DED}^{\mathrm{b}}$ & $\mathrm{DM}$ & Negative (\%) & Suspicious positive (\%) & Positive (\%) & $P$ value \\
\hline \multirow[t]{2}{*}{ Yes } & Yes & 34.5 & 29.9 & 35.6 & 0.015 \\
\hline & No & 36.4 & 45.5 & 18.2 & \\
\hline \multirow[t]{2}{*}{ No } & Yes & 45.0 & 26.1 & 28.8 & 0.004 \\
\hline & No & 56.1 & 33.7 & 10.2 & \\
\hline
\end{tabular}

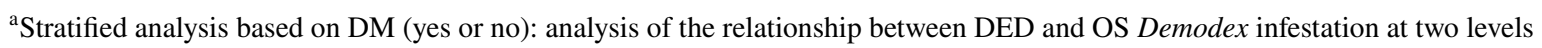

${ }^{\mathrm{b}}$ Stratified analysis based on DED (yes or no): analysis of the relationship between DM and OS Demodex infestation at two levels

Table 3 Comprehensive analysis of the effects of DM and DED on OS Demodex infestation

\begin{tabular}{llllllll}
\hline $\begin{array}{l}\text { OS Demodex } \\
\text { infestation }^{\mathrm{a}}\end{array}$ & $\begin{array}{l}\text { DM+and } \\
\text { DED +(\%) }\end{array}$ & $\begin{array}{l}\text { DM+and } \\
\text { DED- (\%) }\end{array}$ & $\begin{array}{l}\text { DM- and } \\
\text { DED+(\%) }\end{array}$ & $\begin{array}{l}\text { DM- and } \\
\text { DED- (\%) }\end{array}$ & Pearson $\chi^{2}$ test & \multicolumn{2}{l}{$\begin{array}{l}\text { Pearson coef- } \\
\text { ficient }\end{array}$} \\
\hline- & 17.5 & 29.2 & 21.1 & 32.2 & $<0.001$ & $0.197<$ & $<0.001$ \\
\pm & 19.5 & 21.8 & 33.8 & 24.8 & & & \\
+ & 34.1 & 35.2 & 19.8 & 11.0 & & \\
\hline
\end{tabular}

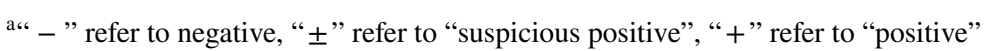

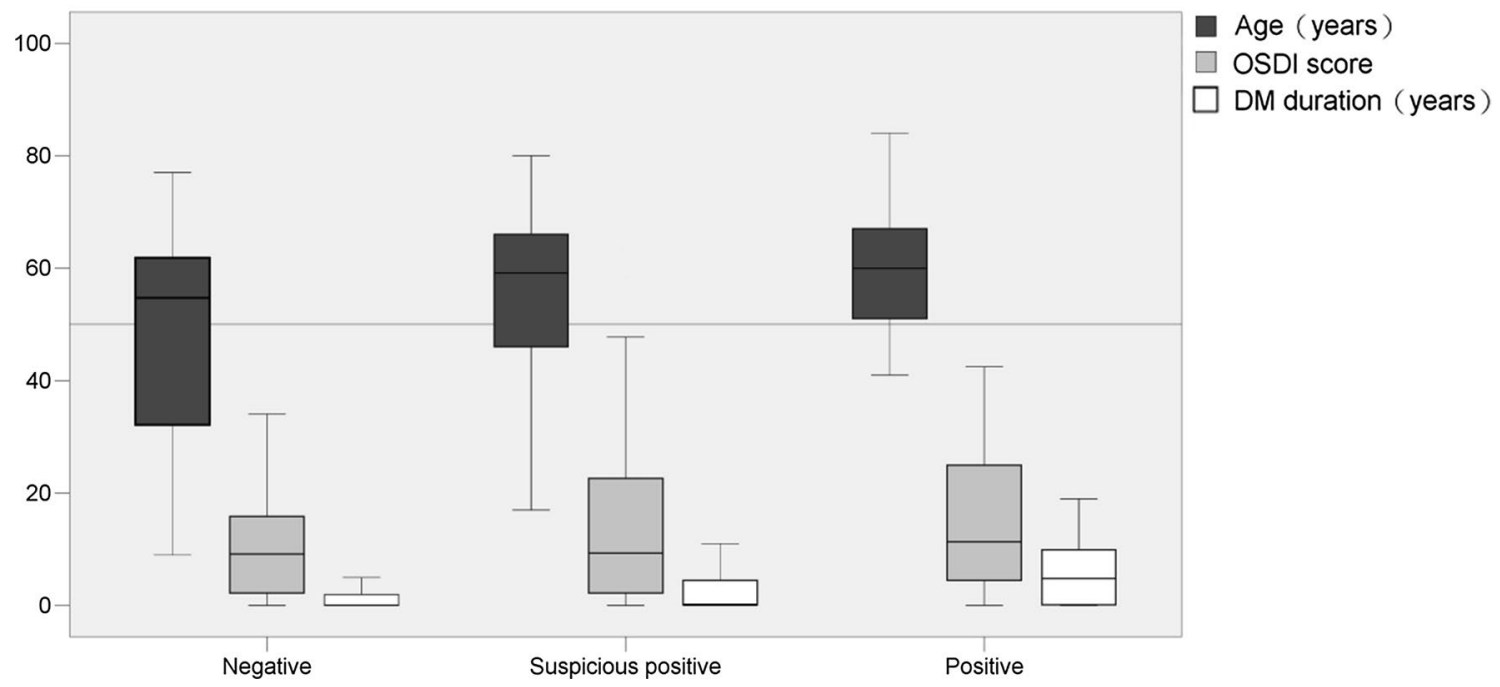

Fig. 2 Further analysis of the age, DM duration, and OSDI scores in the OS Demodex infestation group by Box-plot. Age, OSDI score, and duration of diabetes increased with the severity of Demodex infestation, especially with age and OSDI score

rates increase with age, reaching $84 \%$ in the general population over 60 years of age and $100 \%$ in the general population over 70 years of age [20] There are a number of risk factors that may predispose patients to ocular vermiform, such as rosacea, alcohol intake, smoking, stress, and systemic immunocompromised status [1].
In recent years, some studies have shown that DM, DED, and OS Demodex infestation are related. However, there has not been a study to show whether the two diseases interact in the process of OS Demodex infestaion. To better understand it, we designed this study and demonstrated that DM and DED were both risk factors for OS Demodex infestation, and 
Table 4 Analysis of linear trends between OS Demodex infestation and age, DM duration, and OSDI scores

\begin{tabular}{lrrrrrrrr}
\hline Characteristic & \multicolumn{2}{l}{ Pearson $\chi^{2}$ test } & & \multicolumn{2}{l}{ M-H test } & & \multicolumn{2}{l}{ Pearson coefficient } \\
\cline { 2 - 3 } & $\chi^{2}$ value & $P$ value & & $\chi^{2}$ value & $P$ valule & & $R$ value & $P$ value \\
\hline Age & 63.920 & $<0.001$ & 38.974 & $<0.001$ & & 0.299 & $<0.001$ \\
DM duration(year) & 46.038 & $<0.001$ & 28.701 & $<0.001$ & & 0.264 & $<0.001$ \\
OSDI & 6.459 & 0.596 & 1.558 & & 0.212 & & 0.069 & 0.212 \\
\hline
\end{tabular}

DM duration Diabetic mellitus duration, OSDI Ocular Surface Disease Index specifically uncovered that the association between DM and OS Demodex infestation had precedence over DED. Moreover, we also founded that patients with both diseases had higher risk of OS Demodex infestation.

Here we included 255 participants in our study. However, not all of the patients who participated in this study accepted all the projects involved in this study. For this part of the lost data, we adopt adjacent point interpolation method to make up for it. Fortunately, we found strong associations between OS Demodex infestation and age, MGD, DED, OSDI, DM, DM duration and hypertension. However, no notable correlation was found between OS Demodex infestation with hyperlipidemia. Demodex feeds on the accumulation of skin cells, hormones, and oils in hair follicles, so the gender of the host is thought to be related to the prevalence of ocular Demodex. Besides, Whereas Türk et al. reported that men had a higher rate of Demodex infestation than women [21]. However, our study supports that no discrepancy in OS Demodex infestation between men and women exists and many researchers $[4,5,22]$ had the same results. Serological indicators including HbA1c, HDL, LDL, TG, and TC were also unrelated to OS Demodex infestation, indicating that serological indicators cannot be used as predictors of OS Demodex infestation.

There have been studies on the relationship between ocular surface Demodex infestation and diabetes mellitus. Keskin KR's study found an increase in the density of Demodex in patients with gestational DM [16]. Yamashita LS's found that Demodex infestations were more common in diabetic patients than in healthy volunteers [15]. Our research further certifies that DM is undoubtedly a risk factor of OS Demodex infes tation. DM has an adverse influence on the microvasculature in multiple organs, which aggravates OS Demodex infestation through biochemical pathways involved in facilitating and abrogating microvascular injury and reducing local mucosal reaction, which makes it easier to cause OS Demodex infestation [23].

Meanwhile, the close association between OS Demodex infestation and DED was observed in our research. Randon M's study also suggested that Demodex was implicated in dry eye syndrome, which showed that in symptomatic patients, Demodex infestation was usually associated with
MG dysfunction [19]. Initially, ocular surface Demodex infestations result in dry eye disease. Ocular Demodex can destroy the lacrimal glands and meibomian glands, which causes the reduction of tears and surface oils, leading to dry eye disease. However, because of the immunomodulatory function of tears on the ocular surface, DED causes a decrease in tear secretion, thus impairing the innate immune function of the ocular surface. This can lead to the occurrence of ocular surface Demodex infestation [24]. Overall, OS Demodex infestation and DED interact to jointly worsen ocular surface conditions.

The most essential finding of this study is that the relationship between DM and OS Demodex infestation is significantly greater than that between DED and OS Demodex infestation. After further analysis, we found that patients with both DM and DED had a higher severity of OS Demodex infestation than patients with only one disease, and it was significantly more dangerous than to those with neither disease, which suggests that there is a synergistic effect between DM and DED. The low local immunity of ocular surface may be the key to the interaction between DM and DED to aggravate ocular surface Demodex infestation. The high concentration of blood sugar in the body can cause inflammatory reaction on the ocular surface and damage the normal immune defense mechanism of the body. The occurrence of dry eye disease reduces tear secretion, which further weakens the intrinsic immune function of the ocular surface [23, 24].

Additionally, our study also reveals that age plays an important role in OS Demodex infestation. The analysis suggests a linear positive correlation between age and OS Demodex infestation. Therefore, we suggest that middleaged and elderly people should take more notice of eye hygiene and the cleanliness of their living environment, so as to guard against OS Demodex infestation. Moreover, the prevalence of hypertension is also associated with OS Demodex infestation. The study showed that the severity of OS Demodex infestation in hypertensive patients was significantly higher than in people without hypertension. Vascular disease and micro-vascular hardening caused by hypertension, might resulting in the reduction of local immune function, blocking white blood cells (especially eosinophils), 
and consequently weakening anti-parasitic reactions, which worsens OS Demodex infestation [25].

In conclusion, our study successfully provides evidence that OS Demodex infestation is significantly associated with DM and DED, and DM plays a more significant role than DED in OS Demodex infestation. The severity of OS Demodex infestation in patients with both diseases was significantly higher than that in patients with only one disease and those without any disease. At the same time, age and hypertension have also been identified as risk factors for OS Demodex infestation. Overall, our research not only provides an evidence-based basis for clinical prevention of OS Demodex infestation, but also serves as a big reminder for the majority of people seeking healthy living.

Acknowledgements The authors give all participants credit for their assistance and thank Hong Chen, Li Yang, Lei He, Yanzhen Cheng, and Zhen Zhang in Department of Endocrinology Zhujiang Hospital, Southern Medical University for helping with subject recruitment. This research was supported by Clinical Research Startup Program of Southern Medical University (LC2016YM017) and National Natural Science Foundation of China (No:81800804).

Author Contribution All authors are involved in data collection, input, processing, and analysis.

\section{Declarations}

Conflict of interest None to declare.

Ethical Statements The research has been registered on Chinese clinical trial registry (ChiCTR1800016357). The study was performed in accordance with principles of the Declaration of Helsinki, the International Conference on Harmonisation, Good Clinical Practice guidelines, and all applicable laws and regulations. The study protocol and one amendment to the protocol were reviewed and approved by the Zhujiang Hospital Human Experimental Committee, and all patients provided written informed consent prior to starting study treatment.

Open Access This article is licensed under a Creative Commons Attribution 4.0 International License, which permits use, sharing, adaptation, distribution and reproduction in any medium or format, as long as you give appropriate credit to the original author(s) and the source, provide a link to the Creative Commons licence, and indicate if changes were made. The images or other third party material in this article are included in the article's Creative Commons licence, unless indicated otherwise in a credit line to the material. If material is not included in the article's Creative Commons licence and your intended use is not permitted by statutory regulation or exceeds the permitted use, you will need to obtain permission directly from the copyright holder. To view a copy of this licence, visit http://creativecommons.org/licenses/by/4.0/.

\section{References}

1. Cheng AM, Sheha H, Tseng SC (2015) Recent advances on ocular Demodex infestation. Cur Opi in Ophthalmol 26:295-300. https:// doi.org/10.1097/ICU.0000000000000168

2. Biernat MM, Rusiecka-Ziolkowska J, Piatkowska E, Helemejko I, Biernat P, Gosciniak G (2018) Occurrence of Demodex species in patients with blepharitis and in healthy individuals: a 10-year observational study. Jpn J Ophthalmol 62:628-633. https://doi. org/10.1007/s10384-018-0624-3

3. Hom MM, Schachter SE (2013) Demodex. Optom Vis Sci 90(7):198-205. https://doi.org/10.1097/OPX.0b013e3182968c77

4. Kemal M, Toker MI, Erdoğan H, Topalkara A, Akbulut M (2005) The prevalence of Demodex folliculorum in blepharitis patients and the normal population. Ophthalmic Epidemiol 12:287-290. https://doi.org/10.1080/092865805910057

5. Lee SH, Chun YS, Kim JH, Kim ES, Kim JC (2010) The relationship between Demodex and ocular discomfort. Invest Ophthalmol Vis Sci 51:2906-2911. https://doi.org/10.1167/iovs.09-4850

6. Moris García V, Valenzuela Vargas G, Marín Cornuy M, Aguila Torres P (2019) Ocular demodicosis: a review. Arch Soc Esp Oftalmol 94(7):316-322. https://doi.org/10.1016/j.oftal.2019. 04.003

7. Rather PA, Hassan I (2014) Human demodex mite: the versatile mite of dermatological importance. Indian J Dermatol 59(1):60 66. https://doi.org/10.4103/0019-5154.123498

8. Ben Hadj Salah W, Baudouin C, Doan S, Angoulvant A, Gottlieb J, Bénichou J, Da Cunha E, Eid L, Labetoulle M, Rousseau A (2020) Demodex and ocular surface disease. J Fr Ophtalmol 43(10):1069-1077. https://doi.org/10.1016/j.jfo.2020.08.002

9. Elston CA, Elston DM (2014) Demodex mites. Clin Dermatol 32(6):739-743. https://doi.org/10.1016/j.clindermatol

10. Ahn JM, Lee SH, Rim TH, Park RJ, Yang HS, Kim TI, Yoon KC, Seo KY (2014) Prevalence of and risk factors associated with dry eye: the Korea National Health and Nutrition Examination Survey 2010-2011. Am J Ophthalmol 92:317-325. https://doi. org/10.1016/j.ajo.2014.08.021

11. Gökçe C, Aycankaya Ö, Yula E, Yengil E, Sefil F, Rizaoglu H, Gultepe B, Bayram F (2013) The effect of blood glucose regulation on the presence of opportunistic Demodex folliculorum mites in patients with type 2 diabetes mellitus. J Int Med Res 41:1752-1758. https://doi.org/10.1177/0300060513494730

12. Mongi F, Laconte L, Casero RD (2018) Demodex genus: colonizing parasites of healthy people or mites associated with ocular pathology? Rev Argent Microbiol 50(4):369-373. https://doi.org/ 10.1016/j.ram.2017.09.002

13. Zhang XB, Ding YH, He W (2018) The association between demodex infestation and ocular surface manifestations in meibomian gland dysfunction. Int J Ophthalmol-Chi 11:589-592. https://doi.org/10.18240/ijo.2018.04.08

14. Marathe PH, Gao HX, Close KL (2017) American diabetes association. 2. Classification and diagnosis of diabetes: standards of medical care in diabetes-2019. J Diabetes 9:320. https://doi.org/ $10.2337 / \mathrm{dc} 19-\mathrm{S} 002$ 
15. Toka Özer T, Akyürek Ö, Durmaz S (2020) Association between Demodex folliculorum and metabolic syndrome. J Cosmet Dermatol 19(11):3145-3149. https://doi.org/10.1111/jocd.13721

16. Keskin KR, Aycan KO, Karateke A, Silfeler DB, Soylu KO, Akkoca AN, Hakverdi AU (2014) Increased density of Demodex folliculorum mites in pregnancies with gestational diabetes. Med Princ Pract 23(4):369-372. https://doi.org/10.1159/000363244

17. Jacobi CJ, Paulsen FP, Juenemann AGM (2018) Demodex folliculorum in patients with mild to severe dry eye. Invest Ophth Vis Sci 59:4869. https://doi.org/10.1016/j.oftal.2013.09.003

18. Ayyildiz T, Milletli SF (2020) The effect of ocular demodex colonization on schirmer test and OSDI scores in newly diagnosed dry eye patients. Eye Contact Lens 46(1):S39-S41. https://doi.org/10. 1097/ICL.0000000000000640

19. Randon M, Liang H, El HM, Tahiri R, Batellier L, Denoyer A, Labbã A, Baudouin C (2015) In vivo confocal microscopy as a novel and reliable tool for the diagnosis of Demodex eyelid infestation. Br J Ophthalmol 99:336-341. https://doi.org/10.1136/ bjophthalmol-2014-305671

20. Kabataş N, Sanal DA, Kabataş EU, Acar M, Biçer T, Gürdal C (2017) The effect of Demodex infestation on blepharitis and the ocular symptoms. Eye Contact Lens 43(1):64-67. https://doi.org/ 10.1097/ICL.0000000000000234
21. TãRk M, Oztã Rk I, Sener AG, Kã Ã̃̃KS, AfåAr I, Maden A (2007) Comparison of incidence of Demodex folliculorum on the eyelash follicule in normal people and blepharitis patients. Turkiye Parazitol Derg 31:296-297

22. Koo H, Kim TH, Kim KW, Wee SW, Chun YS, Kim JC (2012) Ocular surface discomfort and demodex: effect of tea tree oil eyelid scrub in demodex blepharitis. J Korean Med Sci 27:15741579. https://doi.org/10.3346/jkms.2012.27.12.1574

23. Kirkman MS, Mahmud H, Korytkowski MT (2018) Intensive blood glucose control and vascular outcomes in patients with type 2 diabetes mellitus. Endocrinol Metab Clin North Am 47:81-96. https://doi.org/10.1016/j.ecl.2017.10.002

24. Sabeti S, Kheirkhah A, Yin J, Dana R (2020) Management of meibomian gland dysfunction: a review. Surv Ophthalmol 65(2):205217. https://doi.org/10.1016/j.survophthal.2019.08.007

25. Agita AM, Alsagaff T (2017) Inflammation, immunity, and hypertension. Acta Med Indones 49:158-165 